\title{
Traumatic Anterior Dislocation of the Knee Without Any Neurovascular Complication - Case Report
}

Nitesh Gahlot $^{1^{*}}$, Uttam Chand Saini ${ }^{2}$ and Smeer Aggarwal ${ }^{1}$

${ }^{1}$ All India Institute of Medical Sciences, Jodhpur, Rajasthan, India

${ }^{2}$ Department of Orthopedics, Post Graduate Institute of Medical Education and Research (PGIMER), Chandigarh, India

"Corresponding author: Nitesh Gahlot, Assistant Professor, AllMS, orthopedics, Jodhpur, Rajasthan 342005, India, Tel: +91 9116096665; E-mail: doc.nitesh@gmail.com

Received date: October 01, 2015; Accepted date: October 16, 2017; Published date: October 23, 2017

Copyright: ( 2017 Gahlot N, et al. This is an open-access article distributed under the terms of the Creative Commons Attribution License, which permits unrestricted use, distribution, and reproduction in any medium, provided the original author and source are credited.

\begin{abstract}
We are reporting a case of traumatic anterior dislocation of the left knee without any neurovascular complication. To our knowledge, such complete dislocation without involvement of popliteal artery and/or peroneal nerve has not been reported before however similar cases are reported after total knee replacement with and without neurovascular compromise. This injury was recognized and treated promptly with rehabilitation commencing early, resulting in a good functional outcome. We discuss the possible injury mechanism and management of this unusual case.
\end{abstract}

Keywords: Knee; Dislocation; Peroneal nerve; Popliteal artery

\section{Introduction}

Acute dislocation of the knee is a limb threatening injury that often results in extensive soft-tissue damage and disruption of the popliteal blood vessels [1-5].

We are reporting a case of an anterior dislocation of the knee in association with disruption of the soft tissues envelop, capsule and anterior cruciate ligament without involvement of popliteal vessels, and the common peroneal nerve. We are not aware of any other reports of such a combination of injuries. However similar cases are reported after total knee replacement with and without neurovascular compromise [6,7].

\section{Case Report}

A 38-year-old man, laborer, suffered a hyperextension injury when his left leg was hit by stones coming down from height. It took the patient $4 \frac{1}{2}$ hours to reach our hospital in an ambulance. His limb had been supported by a Cramer wire splint by the ambulance attendant. On admission, obvious deformity and swelling of the leg were noted; the ankle pulses were well palpable and there was no foot drop with normal sensation in the distribution of the common peroneal nerve. The injury was closed, with no other systems involved. Anteroposterior and lateral radiographs confirmed an anteriorly dislocated knee (Figure 1).

Prompt reduction (around 1 hours after reaching hospital) and immobilization done (Figure 2). The leg was placed in an above-knee back slab. Patient was kept under observation for development of any compartment syndrome or other vascular and neurological symptom for 3 days. MRI done at this time showed grade III tear of ACL and grade II tear of MCL. There was no meniscus injury. Conservative treatment with cast immobilization for MCL was planned according to protocol described by Indelicato P.A [8].
Patient was followed-up at 1 week, 3 weeks, 6 weeks and 3 months and monthly thereafter. At 1 week follow-up slab was converted in cast. This cast was removed at 6 weeks. Clinical examination after removal of this cast showed restricted knee range of motion (0 to 70 degrees) Minimal laxity on varus valgus, anterior drawer and Lachman testing. Patient was put on rehabilitation protocol as describe by Ahn et al. [9] patient showed progressive improvement in knee range of motion and knee stability.

Patient returned to his work at $4^{\text {th }}$ month. There was no pain and limitation of movement at latest follow-up (1 year). Now we are following him up for development of any symptoms of ACL deficiency or progression of osteoarthritis every 3 month interval.

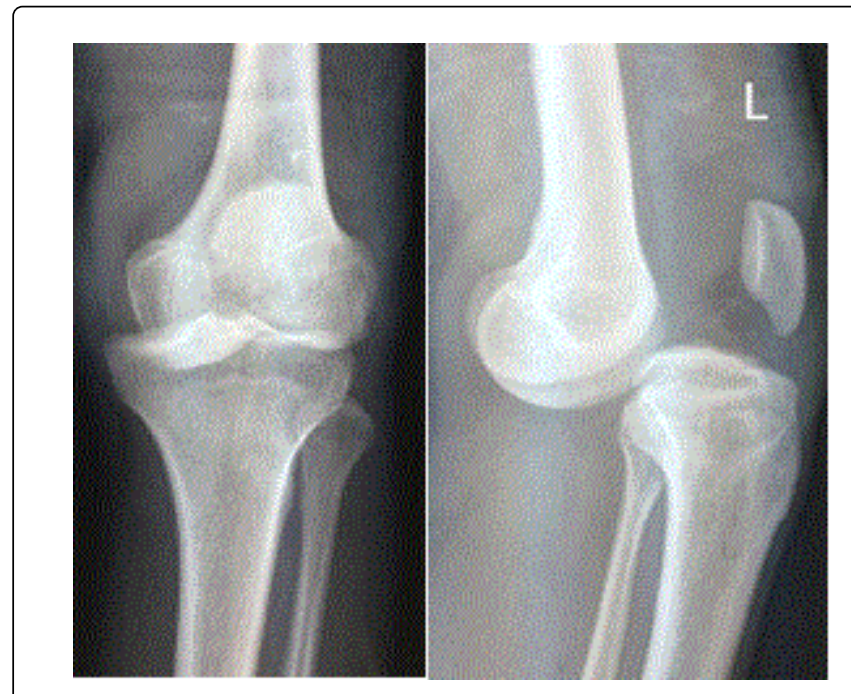

Figure 1: X-Ray AP and Lat view of left knee showing anterior dislocation. 


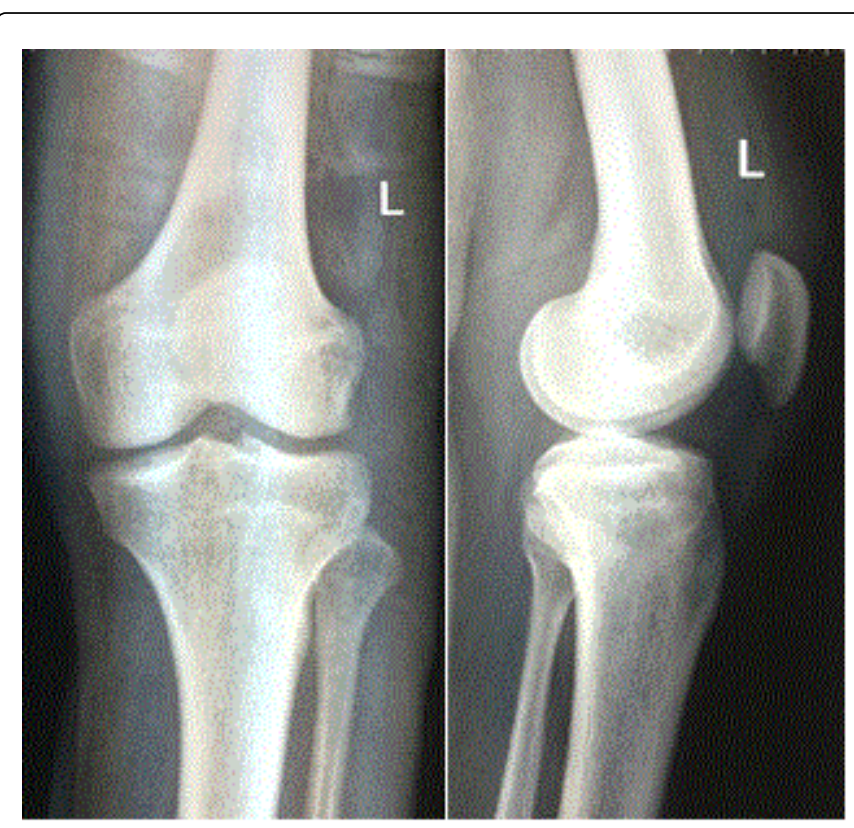

Figure 2: X-Ray AP and Lat view of left knee after reduction of anterior dislocation.

\section{Discussion}

The exact mechanism responsible for knee dislocation with disruption of knee ligaments without popliteal vessels, and the common peroneal nerve was not clear. It was a high-energy blunt injury; the left leg was hit by stones coming down from height. The proximal tibia was then trapped between the stone below and above the leg and patient fall backward with knee went in hyperextension. The momentum caused the knee to hyperextend thus dislocating and disrupting ligaments. Immediate reduction and maintenance of reduced position initially to avoid any neurovascular complication with planned management of the torn ligaments is the internationally accepted treatment of choice.

Prompt recognition of any associated popliteal artery disruption and early revascularization is vital for successful functional results [5,10]. Arteriography is recommended in all cases of knee dislocation with absent or feeble distal pulses. [11].

People who are young and those with sports-related injuries usually have a better prognosis. A retrospective study on a series of 29 acute knee dislocations over a period of 6 years concluded that operative treatment of all the torn structures ensured the best overall knee function with better knee stability and patient satisfaction [12].

Although the differences between early and delayed repair were small, the outcomes in terms of overall knee function, activity levels and anterior tibial translation were better in the knees reconstructed within 2 weeks of injury [13].
In our patient, the injury was severe. Immediate identification and prompt reduction had prevented catastrophic neurovascular complication. Regular follow-up of patient with proper physiotherapy gave good function even with conservative treatment.

It is important to be aware that most common component of such injuries are simultaneous disruption of all soft tissue including nerves, vessels and ligaments, leading to a devastating outcome. Even if vessels and nerves are not involved in initial stage; chances of thrombosis and edema is always there, so immediate identification and prompt reduction is necessary. This case presented the same spectrum of knee ligament disruption, without popliteal artery and common peroneal nerve injuries. This was promptly recognized and thus achieving an eventual good functional result. Such serious injuries require awareness and a team approach to achieve a favorable outcome.

\section{References}

1. Massara M, Prunella R, De Caridi G, Cito D, Serra R, et al. (2017) Traumatic Anterior Knee Dislocation with Popliteal Artery Injury: The Importance of a Prompt Diagnosis and Treatment to Obtain Lower Limb Salvage. Ann Vasc Surg 43: 309e301-309e303.

2. Aydin A, Atmaca H, Muezzinoglu US (2013) Anterior knee dislocation with ipsilateral open tibial shaft fracture: a 5-year clinical follow-up of a professional athlete. Musculoskelet Surg 97: 165-168.

3. Gupta S, Fazal MA, Haddad F (2007) Traumatic anterior knee dislocation and tibial shaft fracture: a case report. J Orthop Surg (Hong Kong) 15: 81-83.

4. Durbhakula SM, Das SP, Uhl RL (2002) Traumatic anterior knee dislocation. Orthopedics 25: 1083-1085.

5. Kennedy JC (1963) Complete Dislocation of the Knee Joint. J Bone Joint Surg Am 45:889-904.

6. Ahn RS, Brown MJ, Santilli MD (2016) Traumatic anterior knee dislocation after total knee arthroplasty. Arthroplasty Today 2: 97-99.

7. Conti A, Camarda L, Mannino S, Milici L, D'Arienzo M (2015) Anterior dislocation in a total knee arthroplasty: A case report and literature review. Journal of orthopaedics 12: S130-132.

8. Indelicato PA (1983) Non-operative treatment of complete tears of the medial collateral ligament of the knee. J Bone Joint Surg Am 65: 323-329.

9. Ahn JH, Chang MJ, Lee YS, Koh KH, Park YS, Eun SS (2010) Nonoperative treatment of ACL rupture with mild instability. Arch Orthop Trauma Surg 130: 1001-1006.

10. Sisto DJ, Warren RF (1985) Complete knee dislocation. A follow-up study of operative treatment. Clin Orthop Relat Res 198: 94-101.

11. Witz M, Witz S, Tobi E, Shnaker A, Lehmann J (2004) Isolated complete popliteal artery rupture associated with knee dislocation. Case reports. Knee Surg Sports Traumatol Arthrosc 12: 3-6.

12. Wong CH, Tan JL, Chang HC, Khin LW, Low CO (2004) Knee dislocations-a retrospective study comparing operative versus closed immobilization treatment outcomes. Knee Surg Sports Traumatol Arthrosc 12: 540-544.

13. Liow RY, McNicholas MJ, Keating JF, Nutton RW (2003) Ligament repair and reconstruction in traumatic dislocation of the knee. J Bone Joint Surg Br 85: 845-851. 